\title{
Corrections to the Capacitance between Two Electrodes Due to the Presence of Quantum Confined System
}

\author{
M. MACUCCI ${ }^{\mathrm{a}^{*}}$ and K. HESS ${ }^{\mathrm{b}}$ \\ ${ }^{\mathrm{a}}$ Dipartimento di Ingegneria dell' Informazione, Via Diotisalvi, 2, I-56126 Pisa, Italy, ${ }^{\mathrm{b}}$ Beckman Institute, $405 \mathrm{~N}$ Mathews, Urbana, IL \\ 61801, USA
}

\begin{abstract}
We have studied the capacitance between two parallel plates enclosing a quantum confined system and its dependence on the applied voltage. The concepts of capacitance and differential capacitance are discussed together with their applicability to systems characterized by single-electron tunneling. We determine the tunneling thresholds by means of a formalism based on the minimization of the system free energy and we retrieve, as a special case, Luryi's quantum capacitance formula. We apply our method to the study of an idealized system made up of a number of quantum dots with random size distributed according to a gaussian. Results are shown for different choices of the position of the dots between the plates and of the voltage span applied to perform the measurement of the differential capacitance.
\end{abstract}

Keywords: quantum dot, capacitance, single-electron, tunneling thresholds

\section{CAPACITANCE AND DIFFERENTIAL CAPACITANCE FOR A QUANTUM SYSTEM}

The capacitance between two electrodes is defined as the ratio of the charge stored on such electrodes to the potential difference between the electrodes. If we consider a system such as that represented in Fig. 1, made up of two plates between which quantum dots are present, the capacitance between the two plates is influenced by the charge status of each dot. In particular, let us assume, here and in the following, that tunneling is possible from the lower plate to the dots but not from the dots to the upper plate. This is true in a wide range of experimental situations, from the classical MOS capacitor, in which the electrons forming the inversion layer come from thermal or optical generation in the semiconductor bulk to several semicon- ductor heterostructures, in which quantum dots are formed on a plane isolated from doped layers by a reasonably transparent tunnel barrier and are charged by applying a suitable voltage to an upper electrode separated by an opaque spacer layer (see e.g. Ref. [1]).

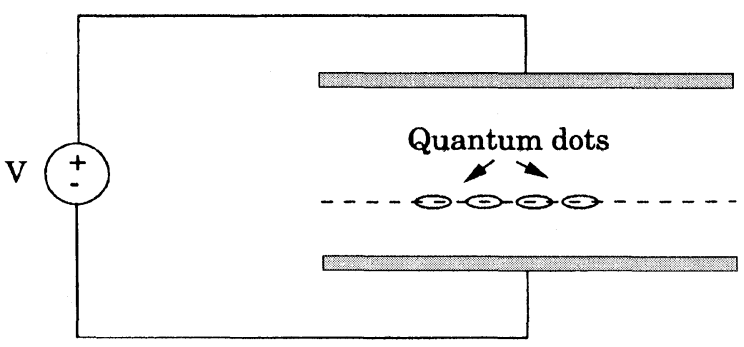

FIGURE 1 System with two parallel plates, held at constant voltage. enclosing a quantum system

* Corresponding author. E-mail: macucci@mercurio.iet.unipi.it 
When an electron tunnels from the lower plate to one of the quantum dots, the charge on the upper plate changes by an amount $\Delta Q=k q$, where $q$ is the electron charge and $k$ is the fraction of the distance between the lower and the upper plate at which the dot is located. This is a consequence of elementary electrostatics and can be obtained, for example, from the application of the Ramo-Shockley theorem [2]. Since the voltage between the two plates is held constant by the voltage source $V$, this is equivalent to a variation in the capacitance measured between the two plates by an amount $\Delta C=k q / V$.

The differential capacitance is instead defined as the limit for $\delta V \rightarrow 0$ of the ratio $\delta Q / \delta V$. If we strictly apply this definition, the differential capacitance $C_{d}$ is equal to the geometrical capacitance $C$. This is because tunneling of each additional electron occurs for a well defined threshold (in the hypothesis of zero temperature), and, for $\delta V \rightarrow 0$, the number of electrons in the dots does not vary during the measurement, unless we hit exactly one of the bias values corresponding to the thresholds. However, real measurements are performed by applying a finite, small alternating voltage to the electrodes, on top of the DC bias. Therefore, if the applied $\delta V$ spans across one or more tunneling thresholds, the variation of the charge stored on the top electrode will be given by

$$
\delta Q=C \delta V+M k q,
$$

where $M$ is the number of tunneling thresholds within $\delta V$. Thus, the measured differential capacitance will be

$$
C_{d}=C+\frac{M k q}{\delta V} .
$$

In the following we shall consider only the second term on the r.h.s. of Eq. (2) and denote it by $C_{d}^{*}$. This quantity is clearly dependent both on the value of $\delta V$ and on the DC bias value.

\section{EVALUATION OF THE TUNNELING THRESHOLDS}

We determine the number of electrons contained in each dot for a given bias voltage by minimizing the free energy of the system. An appropriate expression for the free energy must contain the contribution from the work done by the voltage source that keeps the two plates at a constant voltage, an approach that is common in Coulomb Blockade studies [3,4]. One such expression is given by Stopa [4]:

$$
\begin{aligned}
F(N, V)=\sum_{i} & \varepsilon_{i}-\frac{1}{2} \int d^{3} x \rho_{e l}(\vec{x}) \Phi_{s c}(\vec{x}) \\
& +\frac{1}{2} \int d^{3} x \rho_{i o n}(\vec{x}) \Phi_{s c}(\vec{x})-\frac{1}{2} Q_{u} V,
\end{aligned}
$$

where $N$ is the total number of electrons in the dot, $\varepsilon_{i}$ is the energy eigenvalue for the $i$-th electron, $\rho_{e l}$ is the charge density associated with the electrons, $\Phi_{s c}$ is the self-consistent electrostatic potential. $Q_{u}$ is the total charge on the upper electrode. We stress the fact that this expression is rigorously valid only at zero temperature and is applicable with reasonable approximation only up to temperatures for which $k_{B} T$ ( $k_{B}$ being the Boltzmann constant) is small on the scale of the relevant energies. If we assume the Fermi level in the region supplying electrons as the energy reference (the bottom plate in our simplified model), the number of electrons on the dot for a given applied voltage $V$ can be obtained simply by minimizing $F(N, V)$ with respect to $N$.

Let us apply our formalism to the limiting case of an infinite 2D Electron Gas (2DEG) enclosed between two parallel plates (the MOS capacitor). If we define $C_{u}$ as the geometrical capacitance per unit area between the upper plate and the 2DEG and $C_{l}$ as that between the 2DEG and the lower plate, we have that $k=C_{u} /\left(C_{l}+C_{u}\right)$. Remembering that the quantum confinement energy per unit area in a 2 DEG is $\hbar^{2} \pi N^{2} /\left(2 m^{*}\right)$, we obtain, for the free energy of the system,

$$
\begin{gathered}
F(N, V)=\frac{1}{2 C_{u}}(Q+N q k)^{2}+\frac{1}{2 C_{l}}[Q-(1-k) N q]^{2} \\
+\frac{\hbar^{2} \pi N^{2}}{2 m^{*}}-(Q+N q k) V .
\end{gathered}
$$

From the condition $\partial F(N, V) / \partial N=0$ we obtain the number of electrons in the 2DEG, and, equating the corresponding additional charge present on the top electrode to that expected in the presence of a capaci- 
tance $C_{q}$ in parallel with $C_{l}$, we get $C_{q}=m^{*} q^{2} /\left(\hbar^{2} \pi\right)$, which coincides with Luryi's result[5].

In the case of the MOS inversion layer, it is possible to define an equivalent capacitance, independent of the number of electrons in the layer, because of the quadratic dependence of the quantum confinement energy on the electron number. In general this is not possible: if we consider a quantum dot, for example, the presence of confinement energy will influence the thresholds in a way that must be separately evaluated for each transition.

Nevertheless, in our parallel-plate model with 2D dots it is still possible to take advantage of some simplifications in the expression of the free energy. We notice that, for $2 \mathrm{D}$ dots, the quantum confinement energy does not depend on the electric field in the direction orthogonal to the dot plane and is therefore independent of the voltage applied between the two plates. Furthermore. since the system is equivalent to a capacitor $C_{t}$ (representing the capacitance between the plates in the absence of charge in the quantum dot) in parallel with the series of $C_{u}$ (capacitance between the upper plate and the dot) and $C_{l}$ (capacitance between the dot and the lower plate), it can be shown that the variation of electrostatic energy as a consequence of the addition of one electron onto the dot corresponds simply to that for the same addition performed with both plates kept at zero potential (a rigorous proof of this will be given elsewhere [6]). Thus. the problem is reduced to the calculation of $\Delta E(N)=$ $E(N)-E(N-1)$ (the total energy variation with the inclusion of quantum effects) for a system with both plates grounded. By subtracting from this quantity the work performed by the external voltage sources during the electron addition process, we obtain the free energy variation:

$$
F(N, V)-F(N-1, V)=E(N)-E(N-1)-k q V .
$$

Notice that the only dependence on $V$ is in the third term on the r.h.s., which makes the procedure for the minimization of $F(N, V)$ much simpler. Thus, all we need to evaluate are the energy variations $\Delta E(N)$, which can be computed from the self-consistent calculation described in Sec. 3 by means of Slater's tran- sition rule[7]. The tunneling thresholds are then obtained equating to zero the r.h.s. of Eq. (5).

\section{NUMERICAL PROCEDURE AND RESULTS}

We have studied the contribution $\left(C_{d}^{*}\right)$ of 40 quantum dots with randomly distributed size to the differential capacitance measured between two plates enclosing them. We assume that tunneling is possible only between the lower plate and the dots, that the dots are sparse enough as to prevent any dot-dot interaction and that they are all on the same plane, parallel to those of the plates. The model we use for each dot is 2-dimensional [8], but the self-consistent electrostatic field is computed solving the 3D Poisson equation in the hypothesis of cylindrical symmetry. This is possible because there is no interdot interaction and the plates have an infinite extension. The electron density in the dot is obtained, in the mean field approximation, from the solution of the 2D Schrödinger equation with Dirichlet boundary conditions, assuming a sine squared distribution of the charge along the vertical direction for an interval of $10 \mathrm{~nm}$ (the results show very little dependence on the vertical spread of the charge), corresponding to the ground state in the case of hard-wall confinement. Our Poisson solver can treat the general case of variable permittivity (as long as the boundaries between regions of different permittivity have cylindrical symmetry) but for this calculation we have assumed a constant relative permittivity of 12.9 , corresponding to that of gallium arsenide. The dot radius has a random gaussian distribution with mean value $18 \mathrm{~nm}$ and variance $0.8 \mathrm{~nm}$, while the distance between the plates is $180 \mathrm{~nm}$. We also assume that each dot can accommodate, at most, 10 electrons (in reality this depends on the actual height of the confining potential). The results for $C_{d}^{*}$ are presented in Fig. 2 for $k=0.5$ and in Fig. 3 for $k=$ 0.2 . The voltage span for the measurement is $50 \mathrm{mV}$ in both cases. The dashed curves represent the number of electrons in the dots as a function of the bias voltage. Increasing the DC bias we first observe an increase in $C_{d}^{*}$, while we move towards bias values for which the "density" of tunneling events per 


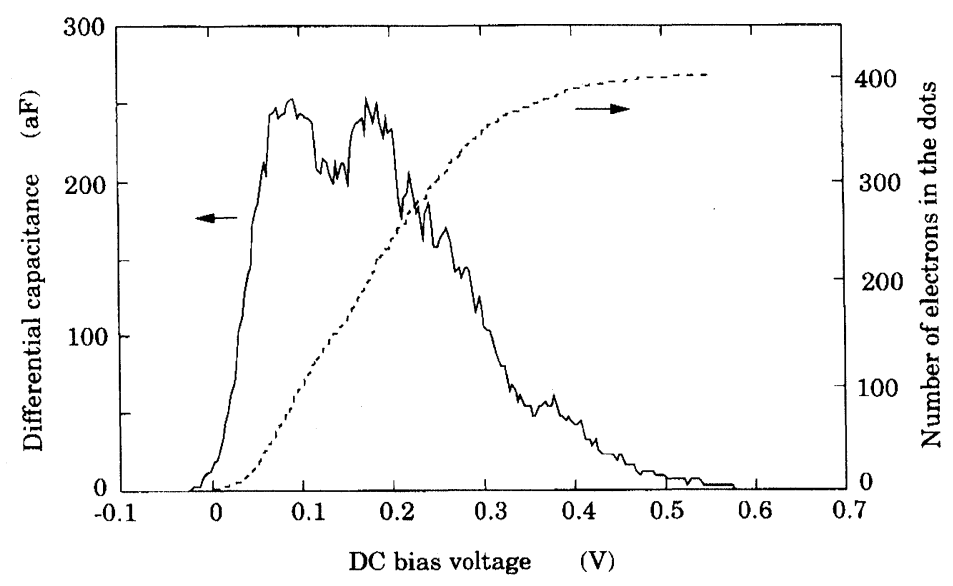

FIGURE 2 Contribution $C_{d}^{*}$ (solid line) to the differential capacitance versus bias voltage for the system described in the text for $k=0.5$ and a voltage span $\delta V=50 \mathrm{mV}$. The dashed line represents the number of electrons in the dots

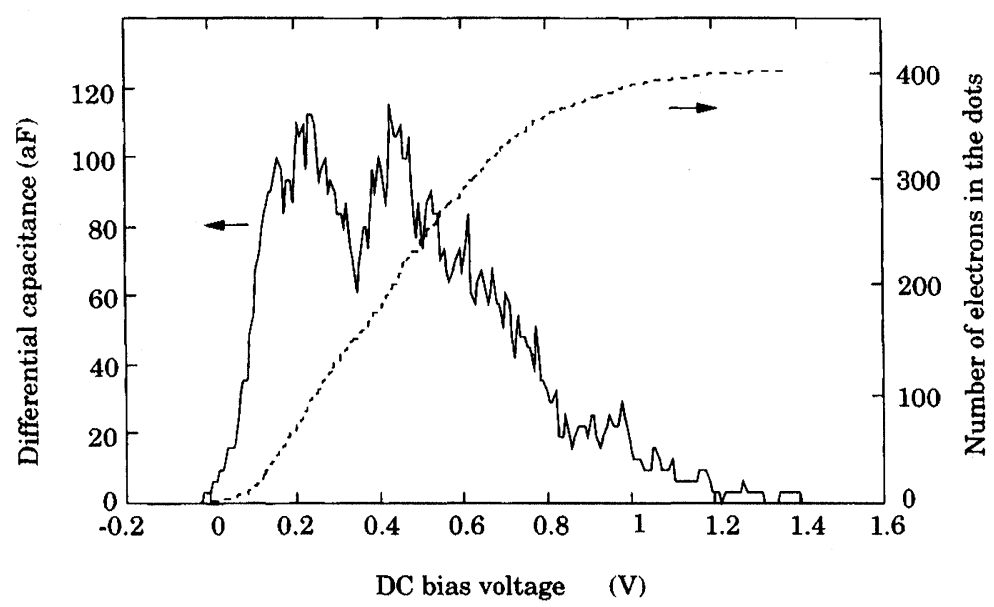

FIGURE 3 Contribution $C_{d}^{*}$ (solid line) to the differential capacitance versus bias voltage for the system described in the text for $k=0.2$ and a voltage span $\delta V=50 \mathrm{mV}$. The dashed line represents the number of electrons in the dots

unit voltage grows. Then, when the dots start saturating, the value of $C_{d}^{*}$ falls down (a 2DEG may start forming, further raising the capacitance. but this is beyond the scope of the present work). The curve is not symmetric because the spacing between the chemical potential values for consecutive numbers of electrons in the dot gets larger for the higher orbitals.

This is just an application to an idealized case, in which the spacing between two consecutive thresholds is much smaller than the voltage span used for the measurement. Other situations are also possible and we are planning to investigate a wide range of them with the more refined model that we are currently developing and which will include a 3D Schrödinger solver. Some quantum capacitance effects, such as those studied in the present paper, may become important also in conventional MOS capacitors if their size is shrunk in the tens of nanometer range. 


\section{Acknowledgements}

This work has received partial support from the NATO Collaborative Research Grant n. 950753. One of the authors (M. M.) acknowledges also support from the Italian Ministry of the University and Scientific Research.

\section{References}

[1] H. Drexler, D. Leonard, W. Hansen, J. P. Kotthaus. P. M. Petroff, "Spectroscopy of quantum levels in charge-tunable InGaAs quantum dots," Phys. Rev. Lett. 73, 2252 (1994).

[2] S. Ramo, "Currents induced by electron motion," Proceedings of the I.R.E., September 1939 ; W. Shockley, "Currents to conductors induced by a moving point charge," J. Appl. Phys. 9, 635 (1938).

[3] J. R. Tucker. "Complementary digital logic based on the Coulomb Blockade," J. Appl. Phys. 72, 4399 (1992).

[4] M. Stopa, "Coulomb oscillation amplitudes and semiconductor quantum-dot self-consistent level structure," Phys. Rev. B 48, 18340 (1993).
[5] S. Luryi. "Quantum capacitance devices," Appl. Phys. Lett. 52, 501 (1988).

[6] M. Macucci and Karl Hess (to be published).

[7] J. C. Slater. The Self-Consistent Field for Molecules and Solids, New York: McGraw-Hill. 1974.

[8] M. Macucci. Karl Hess and G. J. Iafrate, "Simulation of electronic properties and capacitance of quantum dots," J. Appl. Phys. 77, 3267 (1995).

\section{Biographies}

Massimo Macucci is serving on the faculty of the Electrical Engineering Department at the University of Pisa, Italy. His research interests include quantuminterference and single-electron devices, Coulomb Blockade phenomena, modeling and measurements of noise in electron devices.

For the biography of Karl Hess see paper by Grupen and Hess. 

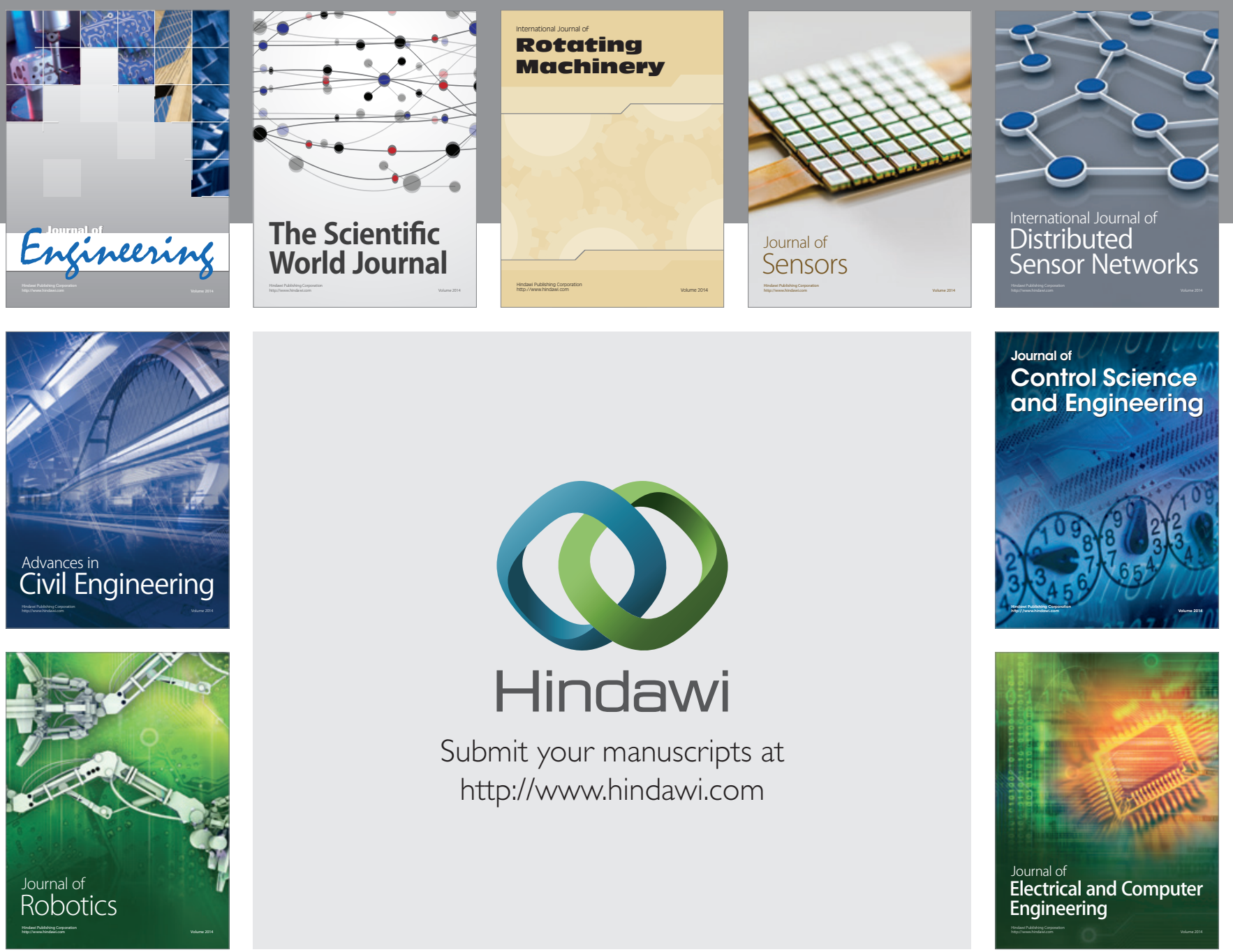

Submit your manuscripts at

http://www.hindawi.com
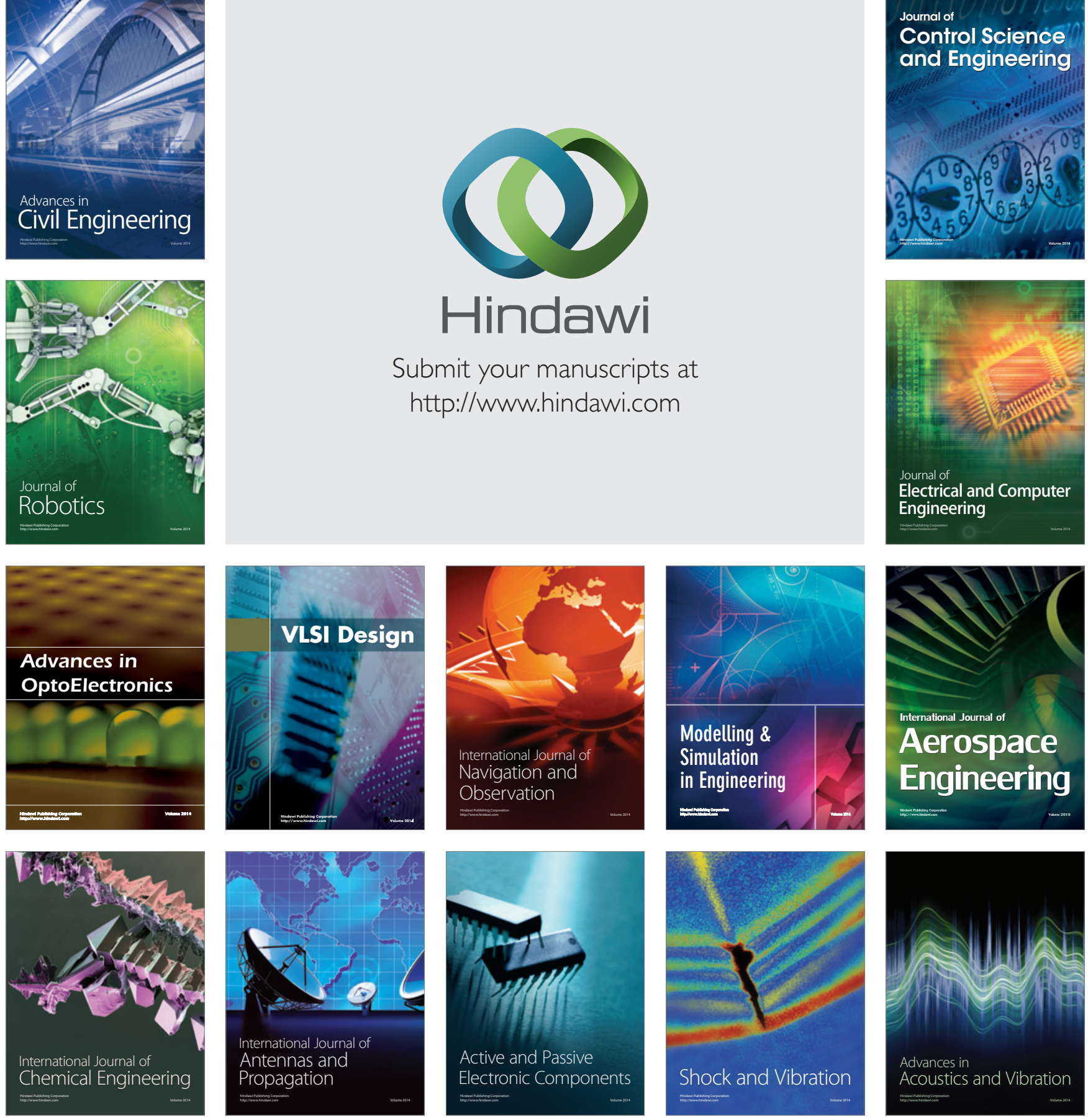